\title{
Conhecimento auditivo da população usuária do Sistema Único de Saúde
}

\section{Unified National Health System users' knowledge about hearing}

\author{
Claudiane José Santana ${ }^{1}$, Patrícia Andrade Bernardino Scopinho ${ }^{2}$, Raquel Santos Ferreira ${ }^{3}$, \\ Taiana de Castro Simões ${ }^{4}$, Juliana Nunes Santos ${ }^{5}$
}

\begin{abstract}
RESUMO
Objetivo: Analisar a percepção, hábitos e conhecimentos auditivos da população usuária do Sistema Único de Saúde das cidades do Vale do Aço. Métodos: Participaram deste estudo, 255 indivíduos distribuídos por Unidades Básicas de Saúde, na faixa etária de 20 a 60 anos. Foram aplicados questionários com 14 perguntas sobre percepção, hábitos e conhecimentos auditivos. Resultados: Em relação às questões de percepção auditiva, foram obtidos os seguintes resultados: 74,9\% escutam bem; 63,1\% têm dificuldade em acompanhar conversa; dor de ouvido (54,5\%); zumbido (46,7\%); plenitude auricular (41,2\%). Com relação a hábitos dos entrevistados, constatou-se: utilizar objetos para limpar o ouvido (80,4\%); ouvir som alto (31,4\%); usar fones de ouvido (7,1\%), julgar prejudicial ouvir som alto (84,7\%); considerar saudável o uso do cotonete (71\%); acreditar na cura da surdez (66,9\%). Encontrou-se relação estatisticamente significativa entre percepção auditiva de não ouvir bem e dificuldade em acompanhar conversa $(\mathrm{p}<0,001)$; presença de zumbido $(\mathrm{p}<0,001)$ e sensação de plenitude auricular $(\mathrm{p}<0,001)$. Dos que referiram não escutar bem, $83,8 \%$ nunca foram submetidos a exame auditivo nem receberam orientação alguma de profissionais da saúde sobre a audição ( $<<0,001)$. Conclusões: Houve grande prevalência de hábitos auditivos prejudiciais, a percepção auditiva dos usuários foi considerável e a população apresentou pouco conhecimento acerca da audição, o que leva a questionar a efetividade das ações de Saúde Auditiva da Atenção Básica propostas pela Portaria 587/2004.
\end{abstract}

Descritores: Audição; Atenção primária à saúde; Centros de saúde; Sistema Único de Saúde

\section{INTRODUÇÃO}

A Organização Mundial de Saúde (OMS) estima que 42 milhões de pessoas acima de três anos de idade são portadoras de algum tipo de deficiência auditiva, de moderada a profunda. Segundo dados da OMS, no Brasil, cerca de 2.250.000 habitantes são portadores de deficiência auditiva, o que corresponderia a $1,5 \%$ da população ocupando o terceiro lugar entre as deficiências no país ${ }^{(1)}$. Elas podem ser de origem congênita ou adquirida e estas últimas podem relacionar-se a maus hábitos

Trabalho realizado na Faculdade de Estudos Administrativos de Minas Gerais - FEAD (MG) - Belo Horizonte (MG), Brasil.

(1) Especializanda em Audiologia pela Faculdade de Estudos Administrativos de Minas Gerais - FEAD (MG) - Belo Horizonte (MG), Brasil.

(2) Especializanda em Audiologia pela Faculdade de Estudos Administrativos de Minas Gerais - FEAD (MG) - Belo Horizonte (MG), Brasil.

(3) Especializanda em Audiologia pela Faculdade de Estudos Administrativos de Minas Gerais - FEAD (MG) - Belo Horizonte (MG), Brasil.

(4) Especializanda em Audiologia pela Faculdade de Estudos Administrativos de Minas Gerais - FEAD (MG) - Belo Horizonte (MG), Brasil.

(5) Mestre; Professora da Faculdade de Estudos Administrativos de Minas Gerais - FEAD (MG) - Belo Horizonte (MG), Brasil.

Endereço para correspondência: Claudiane José Santana. Av. 26 de outubro, 2284, Bela Vista, Ipatinga - MG, CEP 35160-208.

E-mail: claudianesant@yahoo.com.br

Recebido em: 21/11/2007; Aceito em: 5/11/2008 auditivos como: utilizar objetos para limpar a orelha, ouvir som alto e usar fones de ouvido, o que remete à importância de ações promotoras de saúde nessa área a fim de capacitar a população a adquirir hábitos auditivos saudáveis.

Segundo estabelecido na Conferência Internacional de Promoção de Saúde, realizada em Ottawa (1986), promoção da saúde é o processo de capacitação da comunidade para atuar na melhoria de sua qualidade de vida e de saúde, incluindo uma participação no controle desse processo. Um dos grandes objetivos da promoção da saúde é buscar mudanças nas questões referentes à sua preservação. Para tanto, é necessário que a população esteja informada do seu próprio processo saúde/ doença e dos seus direitos sociais. Assim, a promoção da saúde não é exclusiva do setor de saúde, mas atua na direção de um bem-estar global ${ }^{(2)}$.

No Brasil, as ações de promoção, proteção e recuperação da saúde, com a realização integrada das ações assistenciais e das atividades preventivas são de responsabilidade do Sistema Único de Saúde (SUS). E a OMS, assim como o SUS, tem como estratégia a promoção da saúde. O planejamento e a implementação de programas dessas entidades fundamentam-se nas necessidades da população- alvo ${ }^{(3)}$.

Uma das estratégias de promoção da saúde do SUS foi a criação de Redes Estaduais de Atenção à Saúde Auditiva. 
Essas redes são compostas pelas ações de Saúde Auditiva na Atenção Básica, Serviços de Atenção à Saúde Auditiva na Média Complexidade e Serviços de Atenção à Saúde Auditiva na Alta Complexidade.

Quanto às ações de Saúde Auditiva na Atenção Básica ao indivíduo adulto, estas são realizadas por meio das seguintes orientações: evitar acidentes com a introdução de objetos e hastes de limpeza na orelha, que podem ferir e prejudicar a lubrificação natural do conduto (cerúmen); observar fatores de risco para a audição no ambiente de trabalho (ruído, vibração, produtos químicos) e usar equipamentos de proteção individual (EPI); estar atento aos riscos de exposição a ruído nãoocupacional (aparelhos eletrônicos, ambientes de lazer com níveis sonoros elevados e outros); identificar idosos e adultos com queixa de alterações da audição, vertigem, chiado ou zumbido no ouvido que devem ser referenciados para avaliação especializada; orientar e acompanhar o uso de medicamentos para hipertensão, diabetes e problemas renais. Dessa forma, a promoção da Saúde Auditiva na Atenção Básica se dá mediante as seguintes ações: prevenção e identificação precoce de problemas auditivos junto à comunidade; ações informativas e educativas; orientação familiar; encaminhamentos, quando necessário, ao Serviço de Atenção à Saúde Auditiva na Média Complexidade $^{(4)}$.

O Serviço de Atenção à Saúde Auditiva na Média Complexidade constitui a primeira referência para a atenção básica e contra-referência do Serviço de Atenção à Saúde Auditiva na Alta Complexidade. Esse serviço tem como finalidade prestar assistência especializada às pessoas com doenças otológicas $\mathrm{e}$, em especial, às pessoas com deficiência auditiva. Essa assistência compreende: triagem e monitoramento da audição de neonatos, pré-escolares e escolares; diagnóstico de perda auditiva de crianças a partir de três anos de idade, de jovens e de adultos (trabalhadores e idosos), respeitando as especificidades na avaliação exigidas para cada um desses segmentos ${ }^{(4)}$.

Já o Serviço de Atenção à Saúde Auditiva na Alta Complexidade é referência para o diagnóstico das perdas auditivas e sua reabilitação em crianças de até três anos de idade e em pacientes com afecções associadas (neurológicas, psicológicas, síndromes genéticas, cegueira, visão subnormal); para o diagnóstico de perdas unilaterais; e, ainda, para casos em que a realização da avaliação audiológica em serviço de menor complexidade seja difícil. Tem como finalidade prestar assistência multiprofissional especializada às pessoas com doenças otológicas e, em especial, às pessoas com deficiência auditiva. Esse serviço demanda equipamentos para realizar o diagnóstico diferencial das perdas auditivas ${ }^{(4)}$.

Dessa forma, um trabalho eficaz em Saúde Pública deve contemplar um planejamento de ações voltadas às necessidades de um grupo populacional determinado. Para isso, é preciso conhecer as condições de saúde dessa população por meio de levantamentos epidemiológicos.

Em estudo sobre a demanda pelos serviços de Fonoaudiologia realizado do município de Piracicaba, estado de São Paulo (SP) verificou-se a importância de divulgar a intervenção fonoaudiológica e de suas áreas de atuação, especialmente em Audiologia. Desse modo, futuras alterações poderiam ser evitadas $^{(5)}$.
Além disso, um estudo realizado com deficientes auditivos adultos revela que as condutas prejudiciais à audição mais adotadas por esses indivíduos são: frequentar ambiente com som em forte intensidade (37\%), trabalhar em local ruidoso (33\%), não utilizar protetor auricular (10\%), introduzir objetos nos ouvidos $(70 \%)^{(6)}$. Tais condutas poderiam ser evitadas com trabalhos de promoção em saúde objetivando a orientação acerca dos cuidados com a audição.

Vale lembrar que a atuação fonoaudiológica em saúde, desde o início, foi vista como ausência de doença, ou seja, trabalho muito relacionado à reabilitação de alterações já instaladas e, muitas vezes, restrito ao consultório particular. No entanto, com a criação do SUS, muitos profissionais da saúde e, entre eles os fonoaudiólogos, viram-se obrigados a buscar novas formas de atuar e abranger a saúde de toda a população, dando atenção especial à prevenção e à promoção de saúde, garantindo o acesso e a equidade nas ações ${ }^{(7)}$.

Levantamentos epidemiológicos vêm sendo desenvolvidos com o objetivo de adequar as ações da Fonoaudiologia para prevenir ou diagnosticar as alterações fonoaudiológicas presentes na comunidade. Cita-se, nesse caso, uma pesquisa cuja finalidade foi estudar a audição e os hábitos auditivos de jovens de Sorocaba (SP). Os autores dessa pesquisa ressaltaram ser essencial que os fonoaudiólogos, como profissionais de saúde, estejam envolvidos em campanhas de prevenção de acidentes e preservação dos limiares auditivos. Assim, atitudes prejudiciais à audição poderão ser modificadas, preservandose a saúde auditiva e melhorando a própria qualidade de vida do ser humano(8).

Em síntese, acredita-se que ações preventivas na área fonoaudiológica contribuam, significativamente, para promover a saúde geral. Medidas de promoção da saúde relativas à comunicação objetivam eliminar os fatores responsáveis pela origem dos diversos distúrbios fonoaudiológicos decorrentes das questões auditivas ${ }^{(9)}$.

Dessa forma, o objetivo do presente trabalho foi analisar a percepção, os hábitos e conhecimentos auditivos da população de Centros de Saúde da região do Vale do Aço do Estado de Minas Gerais (MG).

\section{MÉTODOS}

O projeto deste trabalho foi aprovado pelo Comitê de Ética e Pesquisa do Hospital Vera Cruz, no parecer número 056/06. Em seguida as cartas de autorização para a realização da pesquisa nas Unidades Básicas de Saúde (UBS) foram encaminhadas aos Secretários de Saúde ou Responsáveis pela Saúde das cidades da região do Vale do Aço (MG).

Trata-se de um estudo transversal descritivo sobre o conhecimento auditivo da população usuária do SUS.

\section{Público alvo e amostragem}

Para a composição das amostras foram selecionadas as cidades de Ipatinga, Coronel Fabriciano e Timóteo (MG). As informações disponíveis sobre a população foram extraídas do banco de dados nacional do SUS ${ }^{(10)}$.

Para o cálculo do tamanho da amostra, considerou-se uma 
Tabela 1. População de acordo com o banco de dados do Sistema Único de Saúde e amostragem estratificada por UBS das cidades de Coronel Fabriciano, Timóteo e Ipatinga

\begin{tabular}{lccccccc}
\hline Local & $\begin{array}{c}\text { População } \\
\text { total }\end{array}$ & $\begin{array}{c}\text { População } \\
\text { SUS }\end{array}$ & $\%$ & $\begin{array}{c}\text { Amostra } \\
\text { inicial }\end{array}$ & $\begin{array}{c}\text { Número } \\
\text { de UBS }\end{array}$ & $\begin{array}{c}\text { Amostra inicial } \\
\text { / UBS }\end{array}$ & $\begin{array}{c}\text { Amostra } \\
\text { final * }\end{array}$ \\
\hline Coronel Fabriciano & 101.511 & 8.199 & 8,08 & 15 & 11 & $1,0=1$ & 15 \\
Timóteo & 76.820 & 31.176 & 40,58 & 57 & 11 & $3,8=4$ & 60 \\
Ipatinga & 225.642 & 94.283 & 41,78 & 174 & 16 & $11,6=12$ & 180 \\
\hline Total & 403.973 & 133.658 & 33,09 & 246 & 38 & -- & 255 \\
\hline
\end{tabular}

Fonte: DATASUS/ 2004

Legenda: UBS = Unidade Básica de Saúde, SUS = Sistema Único de Saúde; * A amostra final foi obtida multiplicando-se o número de UBS a serem visitadas por 15, que é o número de pessoas entrevistadas por UBS

população total de 133.658 indivíduos, nível de significância de $5 \%$, margem de erro de cinco pontos percentuais e proporção do evento na população próxima de $20 \%$.

Foi realizada uma amostragem aleatória estratificada por UBS das três cidades, conforme pode ser visualizado na Tabela 1.

A amostra constou de 255 sujeitos adultos de ambos os sexos, frequentadores dos Centros de Saúde. Foram selecionados indivíduos de 20 a 60 anos de idade, de acordo com classificação do IBGE, referindo-se às ações de atenção à população adulta.

Foram excluídos da pesquisa os indivíduos que não quiseram participar do estudo, e aqueles que não apresentaram capacidade de responder ao questionário.

\section{Coleta de dados}

Após a identificação ao gerente da UBS, foi feito um esclarecimento oral aos usuários que aguardavam atendimento nas Unidades sobre o caráter voluntário da pesquisa, bem como seus objetivos e procedimentos. Em seguida, foi distribuído aos participantes, um termo de consentimento livre e esclarecido, o qual foi devidamente assinado, autorizando a participação no presente estudo.

Posteriormente, foi solicitado aos participantes que respondessem ao questionário (Anexo 1) estruturado pelas autoras do presente trabalho, segundo a Portaria 587 de 07 de outubro de $2004^{(4)}$. O questionário foi elaborado com 14 questões fechadas relativas aos dados pessoais dos participantes (idade, sexo, estado civil, escolaridade, renda familiar); questões relacionadas à percepção auditiva (se a pessoa acha que escuta bem, se tem sensação de dor de ouvido, zumbido, que um ouvido é melhor que o outro, se tem dificuldade de acompanhar conversa, se tem sensação de ouvido abafado); perguntas referentes a hábitos auditivos (utiliza objetos para limpar o ouvido, ouve som alto, usa fones de ouvido); perguntas acerca de conhecimentos auditivos (acha que o uso do cotonete é saudável, que o som alto é prejudicial, que surdez tem cura). A coleta desses dados foi realizada por apenas duas das pesquisadoras para priorizar a padronização das respostas. Durante a aplicação, o pesquisador perguntava e o usuário respondia (entrevista estruturada), assim os participantes tinham liberdade de expressar suas dúvidas e fazerem perguntas que eram esclarecidas. Esse mesmo questionário foi testado com a aplicação de um estudo piloto, com dez usuários, e as questões de difícil entendimento foram reformuladas.
Após a análise dos dados dos questionários, os resultados foram discutidos entre as pesquisadoras e estruturados no programa estatístico EPI INFO 2002. O nível de significância adotado foi de $5 \%(\mathrm{p}<0,05)$.

\section{RESULTADOS}

A presente pesquisa foi realizada com 255 sujeitos adultos na faixa etária de 20 a 60 anos, com idade média de 34,2 anos $( \pm 1,1)$, sendo $192(75,3 \%)$ do sexo feminino e $63(24,7 \%)$ do sexo masculino. A maioria (175-68,6\%) possui primeiro grau completo; $161(63,1 \%)$ são casados; $126(49,4 \%)$ com a profissão predominante de domésticas ou do lar. Apresentam renda familiar variando de um a três salários mínimos (175-68,6\%); residem em casas com média de 4,03 $( \pm 1,7)$ pessoas, sendo todos frequentadores dos Centros de Saúde de três cidades do Vale do Aço (MG): Coronel Fabriciano, Timóteo e Ipatinga.

Os resultados sobre percepção, hábitos e conhecimentos auditivos obtidos nos questionários estão apresentados na Tabela 2 .

Como mostra a Tabela 2, $80 \%$ dos participantes utilizam objetos para a limpeza da orelha. Desses, $8 \%$ utilizam mais de um objeto; $68 \%$ usam o cotonete; $4 \%$ usam outros objetos, como chave, grampo, etc. E $20 \%$ não utilizam objetos para a limpeza, como demonstra a Figura 1.

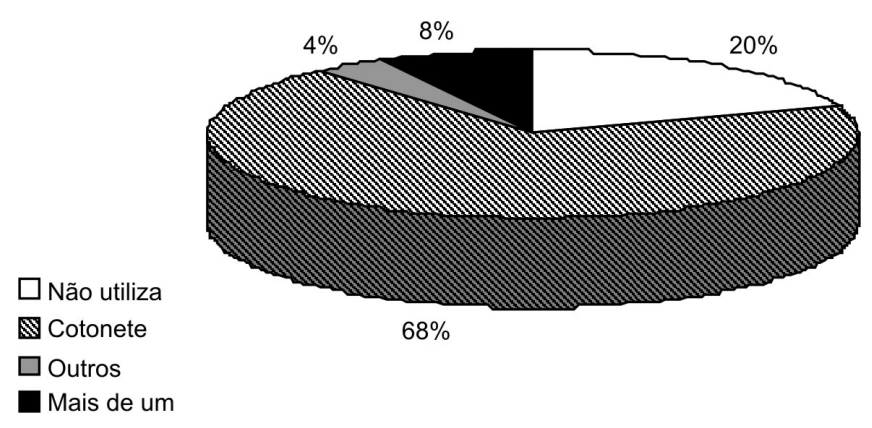

Figura 1. Objetos mais utilizados para limpeza do ouvido

Correlacionando as questões relativas a hábitos auditivos com as questões sobre orientações já recebidas, obteve-se alta incidência de indivíduos que consideram o uso do cotonete saudável. Esses mesmos indivíduos relataram que nunca receberam orientações de profissionais da saúde sobre os cuidados com a audição (relação estatisticamente significante: $\mathrm{p}=0,048)$. 
Tabela 2. Percepção, hábitos e conhecimentos auditivos de 255 usuários do Sistema Único de Saúde nas cidades de Coronel Fabriciano, Timóteo e Ipatinga, abril de 2007

\begin{tabular}{|c|c|c|c|c|}
\hline Questões & $\operatorname{Sim}$ & $\%$ & Não & $\%$ \\
\hline Escuta bem? & 191 & 74,9 & 64 & 25,1 \\
\hline Dor de ouvido? & 139 & 54,5 & 116 & 45,5 \\
\hline Zumbido? & 119 & 46,7 & 136 & 53,3 \\
\hline Ouvido melhor que o outro? & 80 & 31,4 & 175 & 68,6 \\
\hline Dificuldade de acompanhar uma conversa? & 161 & 63,1 & 94 & 36,9 \\
\hline Sensação de ouvido abafado? & 105 & 41,2 & 150 & 58,8 \\
\hline Utiliza objetos para limpar o ouvido? & 205 & 80,4 & 50 & 19,6 \\
\hline Ouve som alto? & 80 & 31,4 & 175 & 68,6 \\
\hline Considera som alto prejudicial? & 216 & 84,7 & 39 & 15,3 \\
\hline Cotonete é saudável? & 181 & 71 & 74 & 29 \\
\hline Usa Fones de ouvido? & 18 & 7,1 & 237 & 92,9 \\
\hline Já recebeu alguma orientação sobre os cuidados com a audição? & 57 & 22,4 & 198 & 77,6 \\
\hline Fez exame ou avaliação auditiva? & 61 & 23,9 & 194 & 76,1 \\
\hline Surdez tem cura? & 152 & 59,6 & 103 & 40,4 \\
\hline
\end{tabular}

Tabela 3. Correlação entre os hábitos e percepções auditivas de 255 usuários do Sistema Único de Saúde nas cidades de Coronel Fabriciano, Timóteo e Ipatinga, abril de 2007

\begin{tabular}{|c|c|c|c|c|c|c|c|}
\hline \multirow{3}{*}{ Características } & & \multicolumn{4}{|c|}{ Escuta bem } & \multirow{3}{*}{ Intervalo de confiança } & \multirow{3}{*}{ Valor de $p$} \\
\hline & & \multicolumn{2}{|c|}{ Sim } & \multicolumn{2}{|c|}{ Não } & & \\
\hline & & $\mathrm{N}$ & $\%$ & $\mathrm{~N}$ & $\%$ & & \\
\hline \multirow{2}{*}{ Dificuldade conversa } & Sim & 105 & 65,2 & 56 & 34,8 & \multirow{2}{*}{$0,07-0,38$} & \multirow{2}{*}{${ }^{*} 0,0007$} \\
\hline & Não & 86 & 91,5 & 08 & 8,5 & & \\
\hline \multirow{2}{*}{ Ouve som alto } & Sim & 60 & 75 & 20 & 25 & \multirow{2}{*}{$0,54-1,85$} & \multirow{2}{*}{0,89} \\
\hline & Não & 131 & 74,9 & 44 & 25,1 & & \\
\hline \multirow{2}{*}{ Tem zumbido } & Sim & 71 & 59,7 & 48 & 40,3 & \multirow{2}{*}{$0,10-0,37$} & \multirow{2}{*}{${ }^{*} 0,000001$} \\
\hline & Não & 120 & 88,2 & 16 & 11,8 & & \\
\hline \multirow{2}{*}{ Ouvido abafado? } & Sim & 62 & 59 & 43 & 41 & \multirow{2}{*}{$0,12-0,42$} & \multirow{2}{*}{${ }^{*} 0,000003$} \\
\hline & Não & 129 & 86 & 21 & 14 & & \\
\hline \multirow{2}{*}{ Usa fones de ouvido? } & Sim & 16 & 88,9 & 02 & 11,1 & \multirow{2}{*}{$0,63-12,68$} & \multirow{2}{*}{0,25} \\
\hline & Não & 175 & 73,8 & 62 & 26,2 & & \\
\hline \multirow{2}{*}{ Fez exame auditivo? } & $\underline{\operatorname{Sim}}$ & 46 & 75,4 & 15 & 24,6 & \multirow{2}{*}{$0,53-2,01$} & \multirow{2}{*}{0,94} \\
\hline & Não & 145 & 74,7 & 49 & 25,3 & & \\
\hline
\end{tabular}

* Correlações significantes $(p<0,05)$ estão assinaladas com asterisco.

A Tabela 3 apresenta a correlação entre os hábitos e percepções auditivas dos 255 indivíduos participantes do presente estudo.

Quanto aos resultados sobre a correlação entre a realização do exame auditivo, orientação profissional e sintomas auditivos, podem ser visualizados na Tabela 4 .

\section{DISCUSSÃO}

Dos participantes desse estudo, $75 \%$ eram do sexo feminino e com renda mensal entre um a três salários mínimos. Esses valores corroboram o encontrado em levantamento epidemiológico dos usuários das UBS do município de Belo Horizonte (MG), no qual verificou-se que a maioria dos entrevistados era do sexo feminino, $32,20 \%$ apresentavam renda mensal entre um e dois salários mínimos e apenas 29,5\% possuíam ensino médio completo ${ }^{(11)}$. Desse modo, percebeu-se que o perfil dos usuários do SUS, que participaram da presente pesquisa, não difere deste estudo. $\mathrm{O}$ fato de a maioria dos participantes pertencer ao sexo feminino pode ser atribuído à prevalência de profissionais do lar $(49,4 \%)$ na amostra, o que se relaciona com maior disponibilidade de tempo para frequentar as UBS.

Nas cidades de Ipatinga, Coronel Fabriciano e Timóteo da Região do Vale do Aço (MG) a percepção auditiva dos sujeitos foi considerável, como mostra a Tabela 2. Assim, 25,1\% referiram não escutar bem e quase metade da população relatou dores de ouvido $(54,5 \%)$, zumbido $(46,7 \%)$ e sensação de ouvido abafado $(41,2 \%)$. Sabe-se que o rebaixamento auditivo traz consigo problemas de ordem emocional e psicossocial, impedindo o indivíduo de desempenhar, plenamente, seu papel 
Tabela 4. Correlação entre a realização de exame auditivo, orientação profissional e sintomas auditivos

\begin{tabular}{|c|c|c|c|c|c|c|c|}
\hline \multirow{3}{*}{ Características } & & \multicolumn{4}{|c|}{ Exame auditivo } & \multirow{3}{*}{ Intervalo de confiança } & \multirow{3}{*}{ Valor de $p$} \\
\hline & & \multicolumn{2}{|c|}{ Sim } & \multicolumn{2}{|c|}{ Não } & & \\
\hline & & $\mathrm{N}$ & $\%$ & $\mathrm{~N}$ & $\%$ & & \\
\hline \multirow{2}{*}{ Dor de ouvido } & Sim & 33 & 23,7 & 106 & 76,3 & \multirow{2}{*}{$0,54-1,74$} & \multirow{2}{*}{0,94} \\
\hline & Não & 28 & 24,1 & 88 & 75,9 & & \\
\hline \multirow{2}{*}{ Zumbido } & Sim & 24 & 20,2 & 95 & 79,8 & \multirow{2}{*}{$0,37-1,21$} & \multirow{2}{*}{0,24} \\
\hline & Não & 37 & 27,2 & 99 & 72,8 & & \\
\hline \multirow{2}{*}{ Ouvido melhor que o outro } & Sim & 22 & 27,5 & 58 & 72,5 & \multirow{2}{*}{$0,72-2,42$} & \multirow{2}{*}{0,45} \\
\hline & Não & 39 & 22,3 & 136 & 77,7 & & \\
\hline \multirow{2}{*}{ Orientação de profissionais } & Sim & 29 & 50,9 & 28 & 49,1 & \multirow{2}{*}{$2,82-10,21$} & \multirow{2}{*}{$>^{*} 0,0001$} \\
\hline & Não & 32 & 16,2 & 166 & 83,8 & & \\
\hline \multirow{2}{*}{ Dificuldade acompanhar conversa } & Sim & 34 & 21,1 & 127 & 78,9 & \multirow{2}{*}{$0,36-1,19$} & \multirow{2}{*}{0,22} \\
\hline & Não & 27 & 28,7 & 67 & 71,3 & & \\
\hline \multirow{2}{*}{ Ouvido abafado? } & $\operatorname{Sim}$ & 26 & 24,8 & 79 & 75,2 & \multirow{2}{*}{$0,60-1,93$} & \multirow{2}{*}{0,90} \\
\hline & Não & 35 & 23,3 & 115 & 76,7 & & \\
\hline
\end{tabular}

* Correlações significantes $(p<0,05)$ estão assinaladas com asterisco.

na sociedade ${ }^{(12)}$. Já dor de ouvido (otalgia) pode ser causada por doenças no próprio ouvido (causa otológica) ou por doenças e/ou distúrbios em outras estruturas orgânicas próximas ou mais distantes do ouvido (causa não-otológica). Quanto ao zumbido, pode ser definido como percepção de som sentido pelos indivíduos, independentemente de estímulo sonoro externo ${ }^{(13)}$. Pessoas que sofrem, ou já sofreram dessa manifestação, apresentam queixas variáveis, algumas delas incomodativas, dificultando as funções mentais normais, tais como raciocínio, memória e concentração. Tais sintomas trazem prejuízos às atividades de trabalho e de lazer, ao repouso e à comunicação nos ambientes social e doméstico ${ }^{(14)}$.

Quanto aos hábitos auditivos, 31,4\% ouvem som alto, apesar de $84,7 \%$ o considerarem prejudicial. Tal dado corrobora a pesquisa sobre hábitos auditivos dos jovens do município de Sorocaba (SP). De acordo com os dados dessa pesquisa, $33,3 \%$ dos indivíduos relataram ouvir som alto ${ }^{(8)}$.

Dos participantes, 7,1\% utilizam os fones de ouvido para ouvir música. Esse resultado diverge do estudo que analisou os hábitos auditivos dos jovens de Sorocaba (SP), no qual, 57,3\% dos indivíduos fazem uso de fones ${ }^{(8)}$. Tal diferença nos resultados pode ser explicada pelo presente trabalho ter incluído não somente indivíduos jovens, mas também na faixa etária de 20 a 60 anos. Ainda sobre o uso de fones de ouvido, outra pesquisa estudou a audição em adolescentes que o utilizavam por um longo período e constatou rebaixamento dos limiares auditivos nessa população ${ }^{(15)}$, além disso, há uma tendência de aumento do número de jovens com limiares audiométricos comprometidos em função da assiduidade de exposição a sons intensos $^{(16)}$. Assim, hábitos auditivos como ouvir som alto e utilizar fones são considerados prejudiciais uma vez que podem alterar a função auditiva, causando impacto negativo à saúde. Embora a música seja considerada um som agradável, quando utilizada de forma intensa torna-se um transtorno na saúde auditiva do indivíduo ${ }^{(17)}$ podendo propiciar a perda auditiva induzida pelo ruído (PAIR) de caráter irreversível.

Com relação ao uso de objetos para limpar a orelha
(80,4\%), 68\% informaram fazer uso do cotonete (Figura 1) e $71 \%$ dos indivíduos consideram saudável o uso desse artigo material. Esse resultado confirma o estudo realizado sobre hábitos auditivos. Tal estudo verificou que $70 \%$ dos entrevistados possuíam o hábito de introduzir objetos nas orelhas. Esse comportamento foi associado ao prurido que, em geral, é decorrente da cera produzida pelas glândulas sebáceas do epitélio da orelha externa. Diversos foram os objetos citados pelos entrevistados, tais como: grampo de cabelo, palitos de fósforo e de dente, cotonete, agulha de tricô, clipes e chave. Um dos riscos desse comportamento é a impactação do cerúmen no canal auditivo, principalmente com o uso do cotonete. Além disso, observa-se que os objetos mencionados apresentam riscos, podendo desencadear lesões como perfuração timpânica ou infecções de orelha média e externa por contaminação dos objetos utilizados ${ }^{(6)}$.

Ao correlacionar os hábitos e percepções auditivas dos usuários do SUS, verificaram-se relações estatisticamente significantes entre zumbido, dificuldade na conversa e plenitude auricular, conforme visualiza a Tabela 3, apresentada anteriormente. Com efeito, dos 255 sujeitos, $34,8 \%$ referiram não ouvir bem e isso interferia na qualidade de suas conversações ( $\mathrm{p}=0,0007$ ). Verificou-se que, dos 40,3\% dos indivíduos que referiram não ouvir bem, associava-se à presença de zumbido $(\mathrm{p}=0,000001)$. Também, $41 \%$ dos entrevistados associaram a plenitude auricular com a percepção auditiva de não escutar bem $(\mathrm{p}=0,000003)$.

Afinal, os dados obtidos demonstraram que os sintomas de possíveis alterações auditivas se fazem presentes na população pesquisada. Dessa forma, orientações e encaminhamentos ao serviço adequado são necessários, pois a educação para a saúde, objetivando mudanças de hábitos, diagnóstico e tratamento precoce, contribui para melhora da qualidade de vida da população. Num estudo sobre hábitos auditivos, ao questionar os indivíduos sobre as medidas tomadas perante a ocorrência de qualquer alteração auditiva, constatou-se que $77 \%$ dos entrevistados informaram procurar um especialista (médico otorrinolaringologista), 
uma vez que têm facilidade de acesso a serviço especializado. Embora tenham recebido atendimentos e orientações da mesma equipe de profissionais, $13 \%$ deles responderam que procuraram por um clínico geral; $7 \%$ por um farmacêutico e $3 \%$ se automedicam. Tais respostas levam a supor que as informações precisam ser transmitidas constante e insistentemente, de maneira que os indivíduos possam assimilá-las até que os hábitos nocivos à audição sejam eliminados ${ }^{(6)}$.

A respeito do conhecimento auditivo dos entrevistados, objeto dessa pesquisa, observou-se que $66,9 \%$ deles julgam que a surdez tem cura. Esse resultado retrata um conhecimento precário sobre a audição e reforça, mais uma vez, a importância da prevenção primária, cujo objetivo é prevenir a ocorrência de doenças e deficiências auditivas ${ }^{(18)}$. Acerca da prevenção, há registro na literatura sobre o trabalho da Fonoaudiologia em saúde pública/coletiva. Essa pesquisa evidenciou que o campo de trabalho relativo ao eixo preventivo-comunitário ou da saúde pública/coletiva configura-se como um espaço privilegiado para o encontro da Fonoaudiologia com a realidade de vida da população brasileira ${ }^{(19)}$. Vale ressaltar que é da competência do fonoaudiólogo desenvolver trabalhos preventivos no que se refere à audição já que essa é umas das áreas de atuação desse profissional ${ }^{(20)}$.

Prosseguindo a análise dos dados da presente pesquisa, verificou-se que queixas auditivas foram apresentadas, mas os sujeitos não têm hábito de procurar serviços de saúde. Esse dado talvez seja explicado pela restrição existente nos postos de assistência do SUS, o que dificulta o acesso dos pacientes a um serviço especializado, já que a demanda é maior que a oferta. Foi verificado ainda que $83,8 \%$ dos entrevistados nunca foram submetidos a um exame auditivo, como também nunca receberam orientação alguma de profissionais da saúde sobre os cuidados com a audição ( $\mathrm{p}=0,0001)$, como se observa na Tabela 4.

A propósito, um estudo sobre o trabalho fonoaudiológico em UBS demonstrou que a presença do serviço de Fonoaudiologia nesses locais contribuiu para maior conhecimento acerca da necessidade da inserção desse profissional na atenção básica, com um perfil voltado à prevenção e promoção da saúde da população ${ }^{(11)}$. De fato, pôde-se constatar, ao observar a deficiência de orientação sobre a audição aos usuários do SUS, que a presença desse profissional se faz necessária nesses locais. Assim, palestras e reuniões dirigidas aos usuários em sala de espera são recursos importantes para maior conscientização e divulgação do direito à saúde e atenção fonoaudiológica voltada para audição ${ }^{(7)}$.

Sobre as orientações recebidas acerca da audição, $77,6 \%$ dos entrevistados relataram não ter recebido informação alguma. Esse resultado se assemelha ao de outra pesquisa que, ao analisar a porcentagem dos sujeitos que participaram de grupos de orientação fonoaudiológica em UBS, no município de Belo Horizonte (MG), verificou-se que 93,2\% deles nunca haviam sido instruídos nos aspectos fonoaudiológicos. Apenas 1,0\% recebeu esclarecimentos acerca dos cuidados com a audição ${ }^{(11)}$. Destaca-se, ainda, nesse estudo, a importância da visão de coletividade que a Fonoaudiologia deve assumir a partir de sua inserção no serviço público, principalmente na atenção básica, porque esta é a porta para o ingresso da população no Sistema de Saúde ${ }^{(21)}$.

Em um estudo realizado em 185 municípios do estado de Pernambuco com o objetivo de pesquisar a oferta de serviços de diagnóstico de deficiência auditiva na média complexidade, observou-se que muito ainda precisa ser feito para que o acesso e oferta do diagnóstico da deficiência auditiva ocorram de forma horizontal, respeitando as diferenças da comunidade através de um modelo universal, integrativo e eficiente como propõe o Sistema Único de Saúde ${ }^{(22)}$.

Conforme os resultados obtidos na presente pesquisa, observa-se, então, que as queixas auditivas e os maus hábitos auditivos se fizeram presentes na vida dos usuários do SUS, demonstrando que as ações de Saúde Auditiva da Atenção Básica, propostas pela Portaria 587/2004, não são efetivadas. Sugere-se que programas de promoção da saúde auditiva e prevenção de suas alterações sejam revistos pelos profissionais responsáveis e implementados nas Unidades Básicas de Saúde. Com efeito, atitudes simples como a informação sobre os cuidados com a audição pode evitar os maus hábitos e prevenir futuros problemas auditivos. Assim, como proposta inicial, sugere-se a confecção de cartazes ilustrativos que realcem os cuidados com a audição, como: realizar a limpeza externa da orelha, não ficar exposto a sons de alta intensidade e sempre procurar um médico quando perceber alguma alteração auditiva.

\section{CONCLUSÃO}

Este estudo demonstrou que $25 \%$ dos entrevistados referiram não escutar bem e quase metade da população relatou dores de ouvido, zumbido e sensação de ouvido abafado. Observou-se grande prevalência de hábitos auditivos prejudiciais à saúde dos usuários do SUS na Região do Vale do Aço - Minas Gerais. Concomitantemente, o conhecimento acerca dos cuidados com a audição foi insuficiente. Além disso, orientações e encaminhamentos aos serviços adequados se mostraram deficientes. Dessa forma, questiona-se a efetividade das ações de Saúde Auditiva da Atenção Básica. Assim sendo, espera-se que os dados desse estudo forneçam subsídios às Secretarias Municipais da região do Vale do Aço (MG) para a criação de programas de promoção de saúde auditiva, em concordância com a Portaria 587 de 07 de outubro de 2004.

Sendo assim, torna-se essencial o investimento nos aspectos preventivos e educativos sobre o assunto, bem como a realização de exames e encaminhamentos àqueles que referiram possuir sintomas que sugerem alguma alteração do sistema auditivo.

Por tudo isso, é de grande importância o envolvimento de profissionais da área da saúde, incluindo fonoaudiólogos, em programas de promoção de saúde. Esses profissionais, com certeza, contribuirão para a educação da população, atuando em prol de sua saúde e qualidade de vida de forma individual e coletiva. 


\begin{abstract}
Purpose: To analyze the perception, habits and knowledge about hearing of a population of Brazilian Unified Health System users at cities from the Vale do Aço region. Methods: Two hundred and fifty five individuals distributed by Primary Care Units and with ages varying from 20 to 60 years participated on the study. They answered a questionnaire that contained 14 questions regarding perception, habits and knowledge about hearing. Results: Regarding the questions about hearing perception, $74.9 \%$ of the subjects reported to have a good hearing; $63.1 \%$ have some difficulty accompanying conversations; $54.5 \%$ have earache, $46.7 \%$ have tinnitus, and $41.2 \%$ have auricular plenitude. Regarding habits, $80.4 \%$ of the subjects reportedly use objects to clean their ears, $31.4 \%$ hear loud music, $7.1 \%$ use earphones, $84.7 \%$ judge that high volumes are harmful; $71 \%$ consider the use of cotton swabs to be healthy, and $66.9 \%$ believe that deafness is treatable. Statistically significant correlations were found between the perception of "bad hearing" and difficulties accompanying conversations $(\mathrm{p}<0.001)$, presence of tinnitus $(\mathrm{p}<0.001)$ and auricular plenitude $(\mathrm{p}<0.001)$. From the subjects that reported not to hear well, $83.8 \%$ were never submitted to hearing tests and had never received orientations from health professionals about hearing abilities $(\mathrm{p}<0.001)$. Conclusions: There was a great prevalence of harmful hearing habits; subjects' hearing perception was considerable, while knowledge about hearing was revealed deficient. These results lead to the questioning of the effectiveness of primary care actions regarding hearing health, as proposed by the Ordinance 567/2004.
\end{abstract}

Keywords: Audition; Primary health care; Health centers; Single health system

\section{REFERÊNCIAS}

1. Silva LPA, Queiros F, Lima I. Fatores etiológicos da deficiência auditiva em crianças e adolescentes de um centro de referência APADA em Salvador - BA. Rev Bras Otorrinolaringol. 2006;72(1):33-6.

2. Brasil. Ministério da Saúde. Carta de Otawa. Brasília: Ministério da Saúde; Projeto Promoção de Saúde, 2001.

3. Brasil. Lei n. 8.080 de 19 de Setembro de 1990 - Dispõe sobre as condições para promoção, proteção e recuperação da saúde, a organização e o funcionamento dos serviços correspondentes e dá outras providências.

4. Brasil. Ministério da Saúde. Secretaria de Atenção à Saude. Portaria SAS/MS 587 de 7 de outubro de 2004 - Determina a organização e a implantação de Redes Estaduais de Atenção à Saúde Auditiva. Brasília (DF): Ministério da Saúde; 2004.

5. Gonçalves CGO, Lacerda CBF, Perotino S, Mugnaine AMM. Demanda pelos serviços de fonoaudiologia no município de Piracicaba: estudo comparativo entre a clínica-escola e o atendimento na Prefeitura Municipal. Pró-Fono. 2000;12(2):61-6.

6. Oliveira JRM, Neuber DRD, Creppe SVTZ, Oliveira VV, Motti TFG. Hábitos que comprometem a conservação da audição residual em deficientes auditivos. J Bras Fonoaudiol. 2005;5(23):375-80.

7. Marin CR, Chun RYS, Silva RC, Fedosse E, Leonelli BS. Promoção da saúde em fonoaudiologia: ações coletivas em equipamentos de saúde e educação. Rev Soc Bras Fonoaudiol. 2003;8(1):35-41.

8. Wazen SRG, Russo ICP. Estudo da audição e dos hábitos auditivos de jovens do município de Sorocaba - SP. Pró-Fono. 2004;16(1):83-94.

9. Gonçalves MS, Tochetto TM, Primo MT. Fonoaudiologia e saúde coletiva: prioridades detectadas pelos usuários de unidades básicas de saúde. Rev Fonoaudiol Brasil. 2005;3(1):1-3.

10. DATASUS/2004[Internet]. Brasília (DF):Datasus; 2004. [citado 2006 Ago 9]. Disponível em: http://w3.datasus.gov.br/datasus/datasus.php

11. Martins CD. Fonoaudiologia e atenção primária: a visão do usuário. Belo Horizonte: Universidade Federal de Minas Gerais; 2005.

12. Creppe SVTZ, Blasca WQ, Oliveira JRM. Análise da efetividade de um programa de adaptação do aparelho de amplificação sonora individual para indivíduos deficientes auditivos idosos. J Bras Fonoaudiol 2005;5(22):284-90.

13. Mendes MH, Morata TC. Exposição profissional à música: uma revisão. Rev Soc Bras Fonoaudiol. 2007;12(1):63-9.

14. Sanches TG, Medeiros IRT, Levy CPD, Ramalho JRO, Bento RF. Zumbido em pacientes com audiometria normal: caracterização clínica e repercussões. Rev Bras Otorrinolaringol. 2005;71(4):427-31.

15. Park JS, Oh SH, Kang PS, Kim CY, Lee KS, Kwang TY, Sakong J. [Effects of the personal stereo system on hearing in adolescents]. J Prev Med Public Health. 2006;39(2):159-64. Korean.

16. Jorge Júnior JJ, Alegre ACM, Greco MC, Angelini MCA, Barros PM. Hábitos e limiares auditivos de jovens em relação à música eletronicamente amplificada em discotecas. Rev Bras Otorrinolaringol. 2001;67(3):297-304.

17. Pfeifeer M, Rocha RLO, Oliveira FR, Frota S. Intercorrência audiológia em músicos após um show de rock. Rev CEFAC. 2007;9(3):423-9.

18. Andrade CRF. Fases e níveis de prevenção em Fonoaudiologia - ações coletivas e individuais. In: Vieira RM, Vieira MM, Avila CRB, Pereira LD, organizadores. Fonoaudiologia e saúde pública. 2a ed. rev e ampl. Carapicuíba: Pró-Fono; 2000. p.81-104.

19. Penteado RZ, Servilha EAM. Fonoaudiologia em saúde pública/coletiva: compreendendo prevenção e o paradigma da promoção da saúde. Distúrb Comun. 2004;16(1):107-16.

20. Andrade AIA, Russo ICP, Lima MLLT, Oliveira LCS. Avaliação auditiva em músicos de frevo e maracatu. Rev Bras Otorrinolaringol. 2002;68(5):714-20.

21. Befi-Lopes DM, organizadora. Fonoaudiologia na atenção primária à saúde. São Paulo: Lovise; 1997.

22. Miranda GMD, Queiroga BAM, Lessa FJD, Leal MC, Caldas Neto SS. Diagnóstico da deficiência auditiva em Pernambuco: oferta de serviços de média complexidade - 2003. Rev Bras Otorrinolaringol. 2006;72(5):581-6. 
Anexo 1. Questionário

Nome:

Data:

D. N.: Idade:

Sexo ( ) feminino ( ) masculino

Estado civil ( ) solteiro (a) ( ) casado (a) ( ) divorciado (a) ( ) viúvo (a)

Escolaridade: ano grau

Número de pessoas que moram na sua casa:

Renda Familiar: ( ) menos de 1 salário mínimo

( ) de 1 a 3 salários mínimos

( ) de 3 a 5 salários mínimos

( ) acima de 5 salários mínimos

Profissão:

1. Você acha que escuta bem?

( ) $\operatorname{sim}$ ( ) não

2. Você utiliza algum objeto para a limpeza do seu ouvido? (cotonete, chave, tampa de caneta ou outros).

( ) $\operatorname{sim}$ ( ) não

Se sim, quais: ( ) cotonete ( ) chave ( ) tampa de caneta ( ) outros

3. Você acha saudável a utilização de cotonete para a limpeza de ouvido?

( ) $\operatorname{sim}$ ( ) não

4. Você já sentiu dor de ouvido?

( ) $\operatorname{sim}$ ( ) não

5. Você tem zumbido no ouvido? (barulho de chuva, apito, cachoeira, grilo, chiado, panela de pressão ou outros).

( ) $\operatorname{sim}$ ( ) não

6. Você tem hábito de ouvir som com volume elevado?

( ) $\operatorname{sim}($ ) não

7. Você considera prejudicial ouvir som alto?

( ) $\operatorname{sim}$ ( ) não

8. Você acha que tem um ouvido melhor que o outro?

( ) $\operatorname{sim}$ ( ) não

9. Você já recebeu alguma orientação de profissionais da saúde sobre os cuidados com a audição?

( ) $\operatorname{sim}($ ) não

10. Você sente dificuldade de acompanhar uma conversa em ambientes ruidosos?

( ) $\operatorname{sim}$ ( ) não

11. Você tem sensação de ouvido abafado?

( ) sim ( ) não

12. Você escuta música com fones de ouvido?

( ) $\operatorname{sim}($ ) não

13. Você já fez algum exame ou avaliação auditiva?

( ) $\operatorname{sim}($ ) não

14. Você acha que a surdez tem cura?

( ) $\operatorname{sim}($ ) não 